\title{
Outgrowth of Rice Tillers Requires Availability of Glutamine in the Basal Portions of Shoots
}

\author{
Miwa Ohashi ${ }^{1,3^{*}} \mathbb{D}$, Keiki Ishiyama ${ }^{1}$, Soichi Kojima ${ }^{1}$, Noriyuki Konishi ${ }^{1,4}$, Kazuhiro Sasaki $^{2}$, Mitsue Miyao', \\ Toshihiko Hayakawa ${ }^{1}$ and Tomoyuki Yamaya ${ }^{1,5}$
}

\begin{abstract}
Background: Our previous studies concluded that metabolic disorder in the basal portions of rice shoots caused by a lack of cytosolic glutamine synthetase $1 ; 2(G S 1 ; 2)$ resulted in a severe reduction in the outgrowth of tillers. Rice mutants lacking GS1;2 (gs 1;2 mutants) showed a remarkable reduction in the contents of both glutamine and asparagine in the basal portions of shoots. In the current study, we attempted to reveal the mechanisms for this decrease in asparagine content using rice mutants lacking either GS1;2 or asparagine synthetase 1 (AS1). The contributions of the availability of glutamine and asparagine to the outgrowth of rice tillers were investigated.

Results: Rice has two AS genes, and the enzymes catalyse asparagine synthesis from glutamine. In the basal portions of rice shoots, expression of OsAS1, the major species in this tissue, was reduced in gs 1;2 mutants, whereas OsAS2 expression was relatively constant. OsAS1 was expressed in phloem companion cells of the nodal vascular anastomoses connected to the axillary bud vasculatures in the basal portions of wild-type shoots, whereas cell-specific expression was markedly reduced in gs 1;2 mutants. OsAS1 was up-regulated significantly by $\mathrm{NH}_{4}{ }^{+}$supply in the wild type but not in $951 ; 2$ mutants. When GS reactions were inhibited by methionine sulfoximine, OsAS1 was up-regulated by glutamine but not by $\mathrm{NH}_{4}{ }^{+}$. The rice mutants lacking AS1 (as1 mutants) showed a decrease in asparagine content in the basal portions of shoots. However, glutamine content and tiller number were less affected by the lack of AS1.

Conclusion: These results indicate that in phloem companion cells of the nodal vascular anastomoses, asparagine synthesis is largely dependent on glutamine or its related metabolite-responsive AS1. Thus, the decrease in glutamine content caused by a lack of GS1;2 is suggested to result in low expression of OsAS1, decreasing asparagine content. However, the availability of asparagine generated from AS1 reactions is apparently less effective for the outgrowth of tillers. With respect to the tiller number and the contents of glutamine and asparagine in gs 1;2 and as 1 mutants, the availability of glutamine rather than asparagine in basal portions of rice shoots may be required for the outgrowth of rice tillers.
\end{abstract}

Keywords: Asparagine, Asparagine synthetase, Cytosolic glutamine synthetase, Glutamine, Tiller, Rice

\footnotetext{
* Correspondence: ohashi@agr.nagoya-u.ac.jp

${ }^{1}$ Graduate School of Agricultural Science, Tohoku University, 468-1 Aoba,

Aramaki-Aza, Aoba-ku, Sendai 980-8572, Japan

${ }^{3}$ Present Address: Graduate School of Bioagricultural Sciences, Nagoya

University, Furo-cho, Chikusa-ku, Nagoya 464-8601, Japan

Full list of author information is available at the end of the article
} 


\section{Background}

Glutamine and asparagine play crucial roles in plant growth and development as the major nitrogen forms for transport via vasculatures from source to sink tissues within various plants (Urquhart and Joy 1981; Ireland and Lea 1999; Lea et al. 2007; Gaufichon et al. 2010). In rice plants, these amides are major nitrogen forms in both phloem (Hayashi and Chino 1990) and xylem sap (Fukumorita and Chino 1982; Funayama et al. 2013; Ohashi et al. 2015a). In higher plants, including rice, glutamine is synthesized by glutamine synthetase (GS) (Lea and Miflin 1974; Yamaya and Oaks 2004), and asparagine is synthesized from glutamine by asparagine synthetase (AS) (Lea et al. 2007; Gaufichon et al. 2010; Ohashi et al. 2015a). Asparagine is catabolized by asparaginase into aspartate as the common precursor of the essential amino acids (Azevedo et al. 2006; Lea et al. 2007; Yabuki et al. 2017).

Rice has three cytosolic GS isoenzymes (GS1, OsGS1;1 - OsGS1;3) and one chloroplastic GS (GS2) (Tabuchi et al. 2007). The occurrence of two AS isoenzymes (OsAS1 and OsAS2) was recently confirmed (Ohashi et al. 2015a). Both GS1;2 and AS1 are important in the primary assimilation of $\mathrm{NH}_{4}{ }^{+}$taken up by rice roots (Funayama et al. 2013; Ohashi et al. 2015a). OsGS1;2 and OsAS1 were specifically accumulated in three cell layers of root surfaces (epidermis, exodermis and sclerenchyma) after $\mathrm{NH}_{4}{ }^{+}$supply to rice roots (Ishiyama et al. 2004; Ohashi et al. 2015a). In both roots and xylem sap after $\mathrm{NH}_{4}{ }^{+}$ supply, rice mutants lacking GS1;2 showed a decrease in glutamine and asparagine content, while mutants lacking AS1 showed a decrease in asparagine content (Funayama et al. 2013; Ohashi et al. 2015a).

Tiller number is a critical agronomic trait defining grain yields in rice and is influenced by the availability of nitrogen (Mae 1997; Sakamoto and Matsuoka 2008; Liu et al. 2011). We found that the lack of GS1;2 suppressed the outgrowth of the tiller axillary bud and hence a substantial decrease in active tiller number and yields (Funayama et al. 2013; Ohashi et al. 2015b). The outgrowth of tiller axillary buds has been proposed to be related to metabolite use efficiency and hormone signalling networks (Domagalska and Leyser 2011; Evers et al. 2011). We recently showed that metabolic disorder in the basal portions of rice shoots lacking GS1;2 caused a severe reduction in the outgrowth of tiller axillary buds, and this reduction was independent of the content of strigolactone (Ohashi et al. 2015b), a phytohormone inhibiting tiller development (Umehara et al. 2008). The basal portions of rice shoots are important organs for the circulation of metabolites because of vascular networks (Hoshikawa 1989). These organs consist of axillary buds, internodes, and shoot apical meristems (SAMs) (Hoshikawa 1989; Ohashi et al. 2015b). In the basal portions of rice shoots, GS1;2 protein localizes in phloem companion cells of the nodal vascular anastomoses connecting to the axillary bud vasculatures (Ohashi et al. 2015b). The lack of GS1;2 caused a large decrease in both glutamine and asparagine contents in roots and the basal portions of shoots (Ohashi et al. 2017). This decrease in glutamine content in the basal portions of shoots caused a deficiency of active cytokinin, which is required for the outgrowth of rice tillers, via the down-regulation of glutamine or its related metabolitedependent cytokinin synthesis (Ohashi et al. 2017). Thus, the availability of glutamine and/or asparagine is required for the outgrowth of rice tillers (Ohashi et al. 2015b, 2017).

In the present study, we focused on the reduction of asparagine content in the basal portions of rice mutants lacking GS1;2 (gs1;2 mutants). Using rice mutants lacking either GS1;2 or AS1 (as1 mutants), we investigated (1) the molecular mechanisms involved in the decrease in asparagine content in the basal portions of $g s 1 ; 2$ shoots and (2) the relation between low availability of asparagine and the reduction in tiller number.

\section{Results}

Reduced Expression of OsAS1 in Phloem Companion Cells of the Nodal Vascular Anastomoses in the Basal Portions of Shoots Lacking GS1;2

Expression of OsAS1 in the basal portions of shoots of $\mathrm{NH}_{4}{ }^{+}$-fed gs 1;2 mutant seedlings at the fourth-leaf stage showed an approximately 60\% reduction compared with the level in the wild-type rice (Fig. 1). However, the expression of OsAS2 in the gs1;2 mutants was less affected in the basal portions (Fig. 1). OsAS1 was expressed at twice the level of OsAS2 in the basal portions of wild-type shoots (Fig. 1).

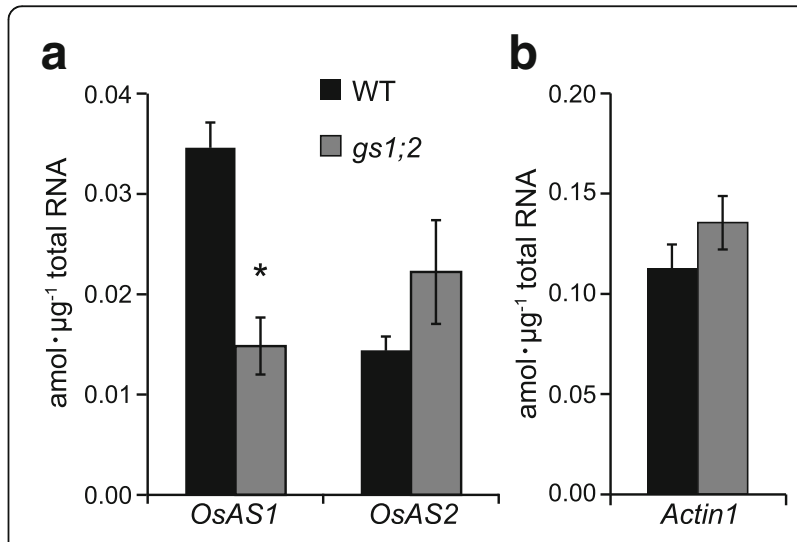

Fig. 1 qPCR analysis of OSASs in the basal portions of wild-type and gs 1;2 mutant shoots. qPCR analysis of OsAS1 and OsAS2 genes (a) and a control Actin 1 (b) in the basal portions of shoots of wild-type plants (WT: black column) and gs 1;2 mutants (gs 1;2: grey column) were performed. Rice seedlings were grown hydroponically in the presence of $1 \mathrm{mM} \mathrm{NH}_{4} \mathrm{Cl}$ until the fourth-leaf stage. Mean values with SE of four independent samples are shown. An asterisk denotes a statistically significant difference between wild type and gs 1;2 mutants ${ }^{*}, P<0.05$ by Student's t-test) 
In situ hybridization revealed strong signals for the OsAS1 transcript in phloem companion cells of the nodal vascular anastomoses in the basal portion of wild-type shoots (Fig. 2d, e). However, the signal intensity for OsAS1 in phloem companion cells of the nodal vascular anastomoses in the gs 1;2 mutants was much lower than in the wild-type rice. Weak signals for OsAS1 were also detected in the SAMs and immature leaves in both the gs1;2 mutants and wildtype rice (Fig. 2a, b, g, h). Signals for the OsAS2 transcript were also detected in phloem companion cells of the nodal vascular anastomoses, and weak signals were detected in the SAM and immature leaves in both the $g s 1 ; 2$ mutants and wild-type rice.
Unlike OsAS1, the signal intensity of the OsAS2 transcript was not significantly different between $g s 1 ; 2$ mutants and wild-type rice (Fig. 3a, b, d, e, g, h). These results fit well with the results obtained from quantitative real-time PCR (qPCR) analysis (Fig. 1). When the control sense probes were used, only background levels of the OsAS1 and OsAS2 transcript were detected (Figs. 2c, f, i, 3c, f, i).

\section{Reduction in OsAS1 Expression and Asparagine Content after $\mathrm{NH}_{4}{ }^{+}$Supply to the gs 1;2 Mutants}

In rice roots, up-regulation of OsAS1 expression and accumulation of asparagine content were observed after $\mathrm{NH}_{4}{ }^{+}$supply (Ohashi et al. 2015a). In the current study,

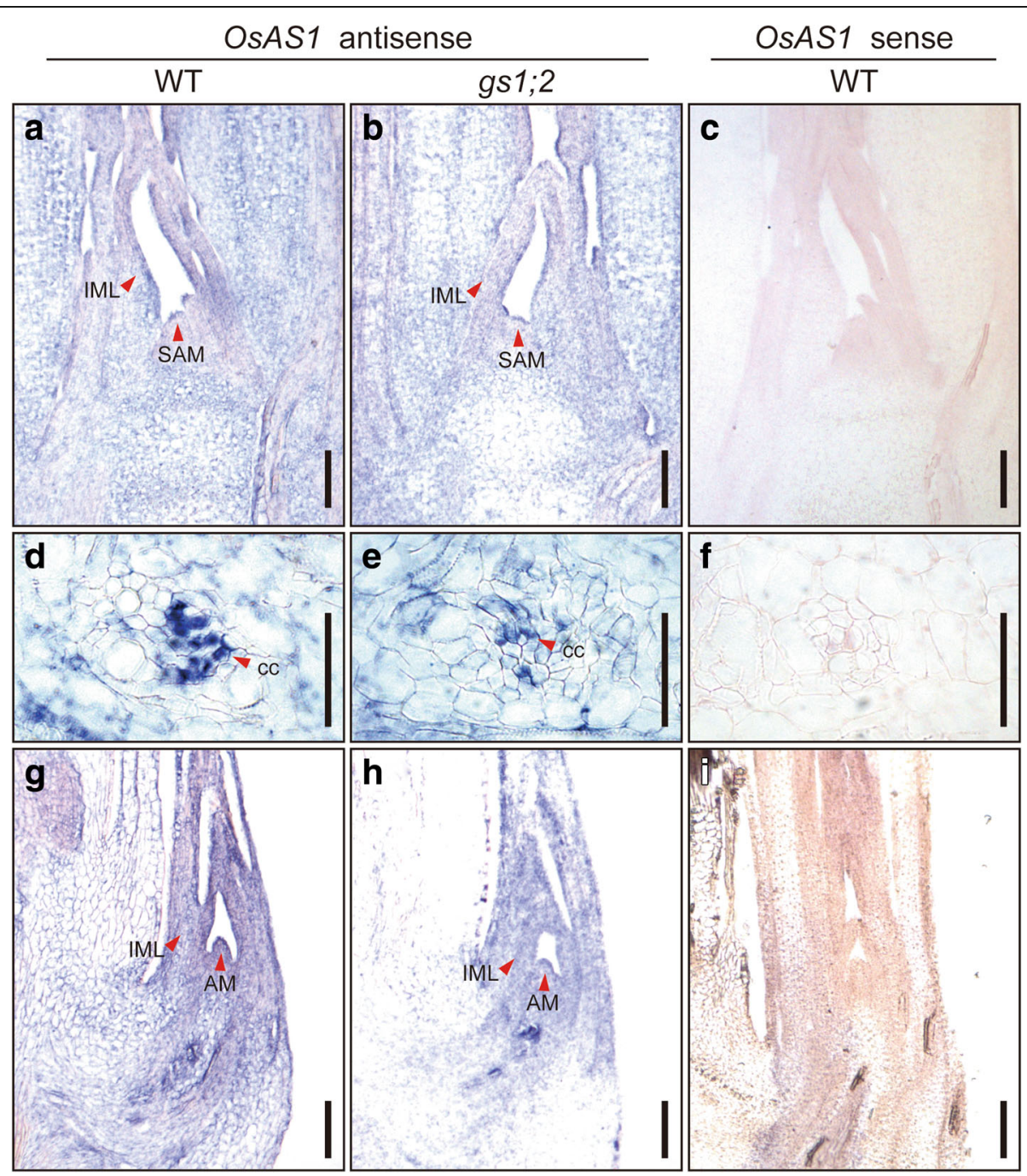

Fig. 2 In situ hybridization for OSAS1 in the basal portions of wild-type and gs 1;2 mutant shoots. Longitudinal sections of basal portions of shoots were prepared from the wild-type rice (WT) $(\mathbf{a}, \mathbf{c}, \mathbf{d}, \mathbf{f}, \mathbf{g}, \mathbf{i})$ and from gs 1;2 mutants (gs 1;2) (b, e, $\mathbf{h})$ grown hydroponically in the presence of 1 mM $\mathrm{NH}_{4} \mathrm{Cl}$ until the fourth-leaf stage. A sense probe was hybridized to WT sections $(\mathbf{c}, \mathbf{f}$, i) as a negative control. Red arrowheads indicate hybridization signals for OSAS1 transcripts in the shoot apical meristem (SAM) (a, b), phloem companion cell of the nodal vascular anastomoses (cc) (d, e), an immature leaf $(\mathrm{IML})$ area $(\mathbf{a}, \mathbf{b}, \mathbf{g}, \mathbf{h})$, and the tiller containing the axillary bud meristem (AM) (g, h). Note that the signal intensity in gs 1;2 (e) was far weaker than in the WT (d). Scale bars $=100 \mu \mathrm{m}(\mathbf{a}-\mathbf{c}, \mathbf{g}-\mathbf{i})$ and $50 \mu \mathrm{m}(\mathbf{d}-\mathbf{f})$ 


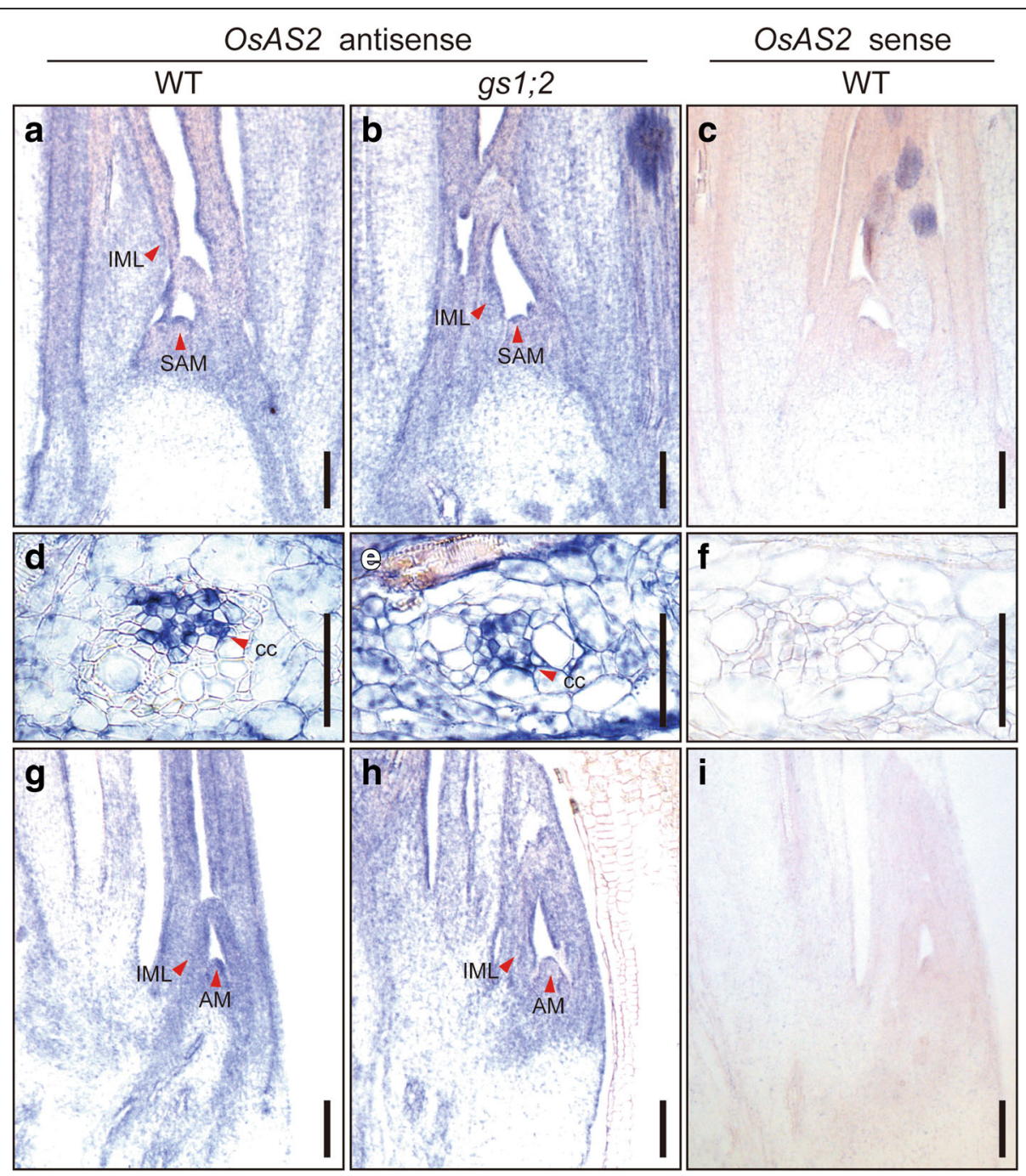

Fig. 3 In situ hybridization for OsAS2 in the basal portions of wild-type and gs 1;2 mutant shoots. Longitudinal sections of basal portions of shoots were prepared from the wild-type rice (WT) $(\mathbf{a}, \mathbf{c}, \mathbf{d}, \mathbf{f}, \mathbf{g}, \mathbf{i})$ and the $g s 1 ; 2$ mutants $(\mathrm{gs} 1 ; 2)(\mathbf{b}, \mathbf{e}, \mathbf{h})$ grown hydroponically in the presence of $1 \mathrm{mM}$ $\mathrm{NH}_{4} \mathrm{Cl}$ until the fourth-leaf stage. A sense probe was hybridized to WT sections $(\mathbf{c}, \mathbf{f}, \mathbf{i})$ as a negative control. Red arrowheads indicate hybridization signals for OsAS2 transcripts in the shoot apical meristem (SAM) (a, b), phloem companion cell of the nodal vascular anastomoses (cc) (d, e), an immature leaf (IML) area $(\mathbf{a}, \mathbf{b}, \mathbf{g}, \mathbf{h})$, and the tiller containing the axillary bud meristem (AM) $(\mathbf{g}, \mathbf{h})$. Scale bars $=100 \mu \mathrm{m}(\mathbf{a}-\mathbf{c}, \mathbf{g}-\mathbf{i})$ and $50 \mu \mathrm{m}(\mathbf{d}-\mathbf{f})$

OsAS1 expression and asparagine content after $\mathrm{NH}_{4}{ }^{+}$ supply were determined in the basal portions of shoots using gs1;2 mutant and wild-type plants. OsAS1 expression was increased over twofold at $8 \mathrm{~h}$ after $\mathrm{NH}_{4}^{+}$ supply in the shoot basal portions of the wild type, whereas it was less changed in the gs1;2 mutants (Fig. 4a). In contrast, there were no significant differences in the expression of OsAS2 and Actin1 between the shoot basal portions of wild type and $g s 1 ; 2$ mutants treated with or without $\mathrm{NH}_{4}{ }^{+}$(Fig. 4b, c). Asparagine was accumulated remarkably at $24 \mathrm{~h}$ after $\mathrm{NH}_{4}{ }^{+}$supply in the shoot basal portions of the wild type, whereas it was relatively constant in the gs1;2 mutants (Fig. 4d). $\mathrm{NH}_{4}{ }^{+}$was highly accumulated in these tissues in $g s 1 ; 2$ mutants after $\mathrm{NH}_{4}{ }^{+}$ supply (Fig. 4e), indicating impairment of $\mathrm{NH}_{4}{ }^{+}$ assimilation. Under the same conditions, glutamine content was less affected $24 \mathrm{~h}$ after $\mathrm{NH}_{4}{ }^{+}$supply in the shoot basal portions of $g s 1 ; 2$ mutants (Ohashi et al. 2017), as in the case of asparagine content in the current study (Fig. 4d).

Further qPCR analysis was carried out in the presence or absence of $1 \mathrm{mM}$ methionine sulfoximine (MSX), an inhibitor of GS reactions. As shown in Fig. 4a, a substantial increase in OsAS1 expression was observed at $8 \mathrm{~h}$ after $\mathrm{NH}_{4}{ }^{+}$supply in the basal portions of shoots, but this increase was not observed when MSX was treated prior to $\mathrm{NH}_{4}{ }^{+}$supply (Fig. 5a). Over a three-fold increase in OsAS1 expression was observed after glutamine supply with or without MSX pre-treatment (Fig. 5a). As in OsAS1 expression, the significant increase in OsGS1;2 expression was 

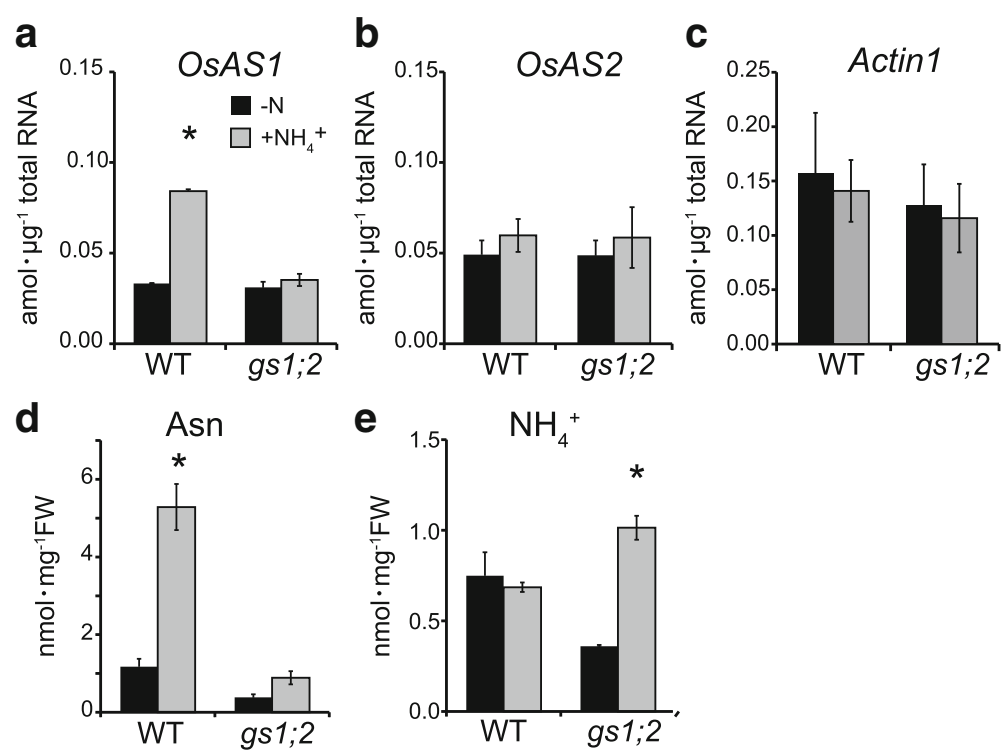

Fig. 4 Expression levels of OsASs and contents of asparagine and $\mathrm{NH}_{4}{ }^{+}$in the basal portions. a - c Transcript contents of the OsAS1 (a), OsAS2 (b) and a control Actin1 (c) in the basal portions of shoots of wild-type plants (WT: black column) and gs 1;2 mutants (gs 1;2: grey column) at the seventh-leaf stage for seedlings grown in water for $3 \mathrm{~d}$ followed by treatments with $\left(+\mathrm{NH}_{4}^{+}\right)$or without (-N) $1 \mathrm{mM} \mathrm{NH}{ }_{4} \mathrm{Cl}$ for $8 \mathrm{~h}$. $(\mathbf{d}$, e) Contents of asparagine (d) and $\mathrm{NH}_{4}{ }^{+}$(e) in the basal portions of shoots of WT (black column) and gs 1;2 (grey column) at the fourth-leaf stage for seedlings grown in water for $3 \mathrm{~d}$ followed by treatments with or without $1 \mathrm{mM} \mathrm{NH}{ }_{4} \mathrm{Cl}$ for $24 \mathrm{~h}$. Mean values with SE of four independent samples are shown. Asterisks denote statistically significant differences between samples treated with and without $1 \mathrm{mM} \mathrm{NH}_{4} \mathrm{Cl}\left({ }^{*}, P<0.05\right.$ by Student's $t$-test)

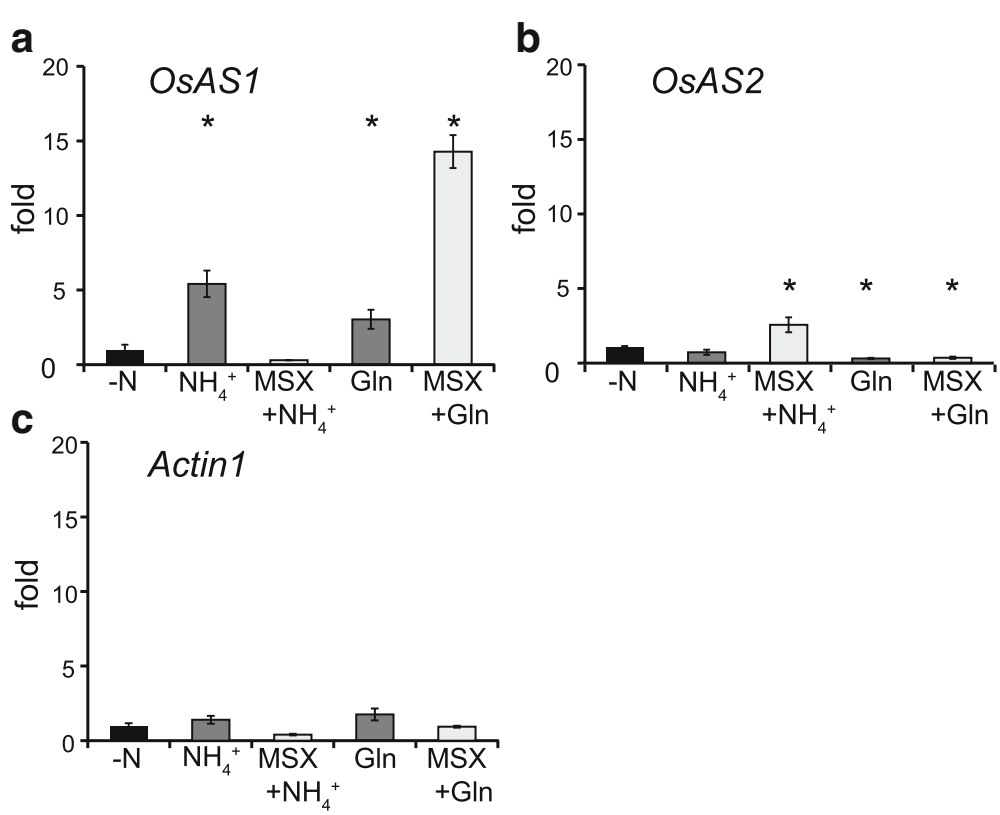

Fig. 5 qPCR analysis of OsASs in the basal portions of wild-type shoots using MSX. Wild-type rice seedlings were grown hydroponically in the presence of $1 \mathrm{mM} \mathrm{NH}_{4} \mathrm{Cl}$ until the fourth-leaf stage and then transferred into water for $3 \mathrm{~d}$. After pre-treatment with $1 \mathrm{mM}$ MSX for $2 \mathrm{~h}$, the seedlings were treated for $8 \mathrm{~h}$ with $1 \mathrm{mM} \mathrm{NH}_{4}{ }^{+}\left(\mathrm{MSX}+\mathrm{NH}_{4}{ }^{+}\right)$or $5 \mathrm{mM}$ glutamine (MSX $\left.+\mathrm{Gln}\right)$. The seedlings without MSX pre-treatment were also treated for $8 \mathrm{~h}$ with $1 \mathrm{mM} \mathrm{NH}_{4}{ }^{+}\left(+\mathrm{NH}_{4}{ }^{+}\right), 5 \mathrm{mM}$ glutamine (Gln) or without nitrogen nutrients (-N). qPCR analyses of OsAS1 (a), OsAS2 (b), and actin 1 transcripts $(\mathbf{c})$ were performed in the shoot basal parts. Fold changes of transcript levels in each treatment relative to those in $-\mathrm{N}$ were calculated, and mean values with SE of four independent samples are shown. Asterisks denote statistically significant differences between the samples treated without nitrogen nutrients $(-N)$ and each treated sample ${ }^{*}, P<0.05$ by Student's $t$-test) 
observed after $\mathrm{NH}_{4}{ }^{+}$or glutamine supply, but an increase in OsGS1;2 expression was not observed for the MSX treatment (Additional file 1: Figure S1b). The expression of OsAS2 was stable after $\mathrm{NH}_{4}{ }^{+}$supply, while glutamine supply caused a decrease in its expression (Fig. 5b). There were slight fluctuations in the expression of OsGS1;1 and Actin1 used as a control treated with or without $\mathrm{NH}_{4}{ }^{+}$ (Fig. 5c, Additional file 1: Figure S1a).

In the roots, OsAS1 expression was increased significantly after $\mathrm{NH}_{4}{ }^{+}$supply, but this increase was not observed in the MSX treatment (Additional file 2: Figure S2a). Increases in OsAS1 expression were observed after glutamine supply with or without MSX treatment (Additional file 2: Figure S2a).

Lack of AS1 Caused a Reduction in Asparagine Content in the Basal Portions of Rice Shoots but not in Tiller Number To examine the contribution of availability of asparagine generated from AS1 reactions in the basal portions of shoots to the outgrowth of rice tillers, reverse genetics studies were carried out using homozygous rice mutants lacking OsAS1 (Ohashi et al. 2015a). Our previous study showed that the lack of AS1 did not affect the expression of other genes related to nitrogen metabolism in roots (Ohashi et al. 2015a). In the basal portions of shoots growing with $\mathrm{NH}_{4}^{+}$, the as1 mutants exhibited no significant difference in OsAS2 expression, but an approximately $80 \%$ decrease in asparagine content was observed compared with the content in the wild type (Fig. 6a, c). In addition, transient $\mathrm{NH}_{4}{ }^{+}$supply for $24 \mathrm{~h}$ to nitrogen-depleted as1 mutants caused an approximately $60 \%$ decrease in asparagine content in the shoot basal portions (Additional file 3: Figure S3a). There were slight fluctuations in the content of glutamine and $\mathrm{NH}_{4}{ }^{+}$ between the basal portions of shoots of as 1 mutants and the wild type when plants were grown with $\mathrm{NH}_{4}{ }^{+}$(Fig. 6d, c, Additional file 3: Figure S3b, d).

No significant differences between as1 mutants and the wild type were observed with respect to tiller number and leaf stage under $1 \mathrm{mM}$ and $2 \mathrm{mM} \mathrm{NH}_{4}^{+}$(Fig. 7). The outgrowth of primary tiller was little affected in as1

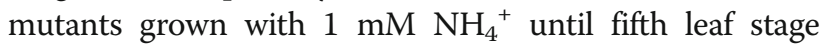
(Additional file 4: Figure S4). The same is true when those plants were grown in the paddy field under normal fertilization (Table 1). There were also no significant differences in shoot length, dry weight, or panicle number between as 1 mutants and wild-type plants grown in the paddy field (Table 1). In our experiments in the paddy field, the lodging phenotype was not observed in as1 mutants (Additional file 5: Figure S5).

Wild-type plants and two lines of as1 mutants (as1-m1 and as $1-m 2$ ) were grown in the paddy field under normal fertilization. Tiller number was measured at the panicle initiation stage. After panicle ripening, rice plants were harvested and shoot length, shoot dry weight without panicle and panicle number were measured. Mean values with SE of four independent plots (five average plants selected from each plot) are shown. One-way ANOVA followed by Bonferroni tests were used to identify significant differences between the wild type and as 1 mutants $(P<0.05)$.

\section{Discussion}

A lack of GS1;2 caused a reduction in asparagine content in the basal portions of shoots (Ohashi et al. 2017). The current study showed that the expression of OsAS1, the major species in the basal portions of shoots, was decreased by the lack of GS1;2, whereas OsAS2, which is dominant in rice leaves, was less affected (Fig. 1) (Ohashi et al. 2015a). In fact, the lack of AS1 severely decreased the asparagine content in the basal portions of shoots (Fig. 6c). These results indicate that the low expression of OsAS1 caused a decrease in asparagine content in the shoot basal portions of $g s 1 ; 2$ mutants. In the basal portions of shoots, GS1;2 and AS1 are suggested to be important in the biosynthesis of asparagine, whereas the contribution of AS2 to this process may be limited (Fig. 1).

Gene expression analyses clearly showed that the expression of OsAS1, but not OsAS2, in both roots and shoot basal portions was enhanced by $\mathrm{NH}_{4}{ }^{+}$and glutamine (Figs. 4, 5, Additional file 2: Figure S2) (Ohashi et al. 2015a). The $\mathrm{NH}_{4}{ }^{+}$-induced OsAS1 expression was inhibited when GS1;2 was missing or GS activity was inhibited by MSX treatment (Figs. 4, 5, Additional file 2: Figure S2). In addition, OsAS1 expression was enhanced by glutamine when GS activity was inhibited by MSX treatment. These results are in close agreement with a previous study by Kawachi et al. (2002), which showed that the $\mathrm{NH}_{4}{ }^{+}$-induced accumulation of AS protein in rice roots was completely inhibited by MSX treatment. These results indicate that glutamine or its related metabolite, but not $\mathrm{NH}_{4}{ }^{+}$itself, acts as a signal molecule for the stimulation of OsAS1 expression in both roots and shoot basal portions, where AS1 is the major isoform (Fig. 5, Additional file 2: Figure S2) (Ohashi et al. 2015a). In addition, the expression of OsAS1 in phloem companion cells of nodal vascular anastomoses, overlapping with OsGS1;2 expression (Ohashi et al. 2015b), was reduced by the lack of GS1;2 (Fig. 2d, e). These results possibly allow glutamine, which is produced by GS1;2, to directly induce the expression of OsAS1 and to be used for asparagine synthesis within these cells. We conclude that the low availability of glutamine caused reduced expression of OsAS1, decreasing asparagine synthesis, in phloem companion cells of the nodal vascular anastomoses in the basal portions of shoots in the gs 1;2 mutants.

There are also several genes reported to be up-regulated by glutamine or related metabolites in rice and other 

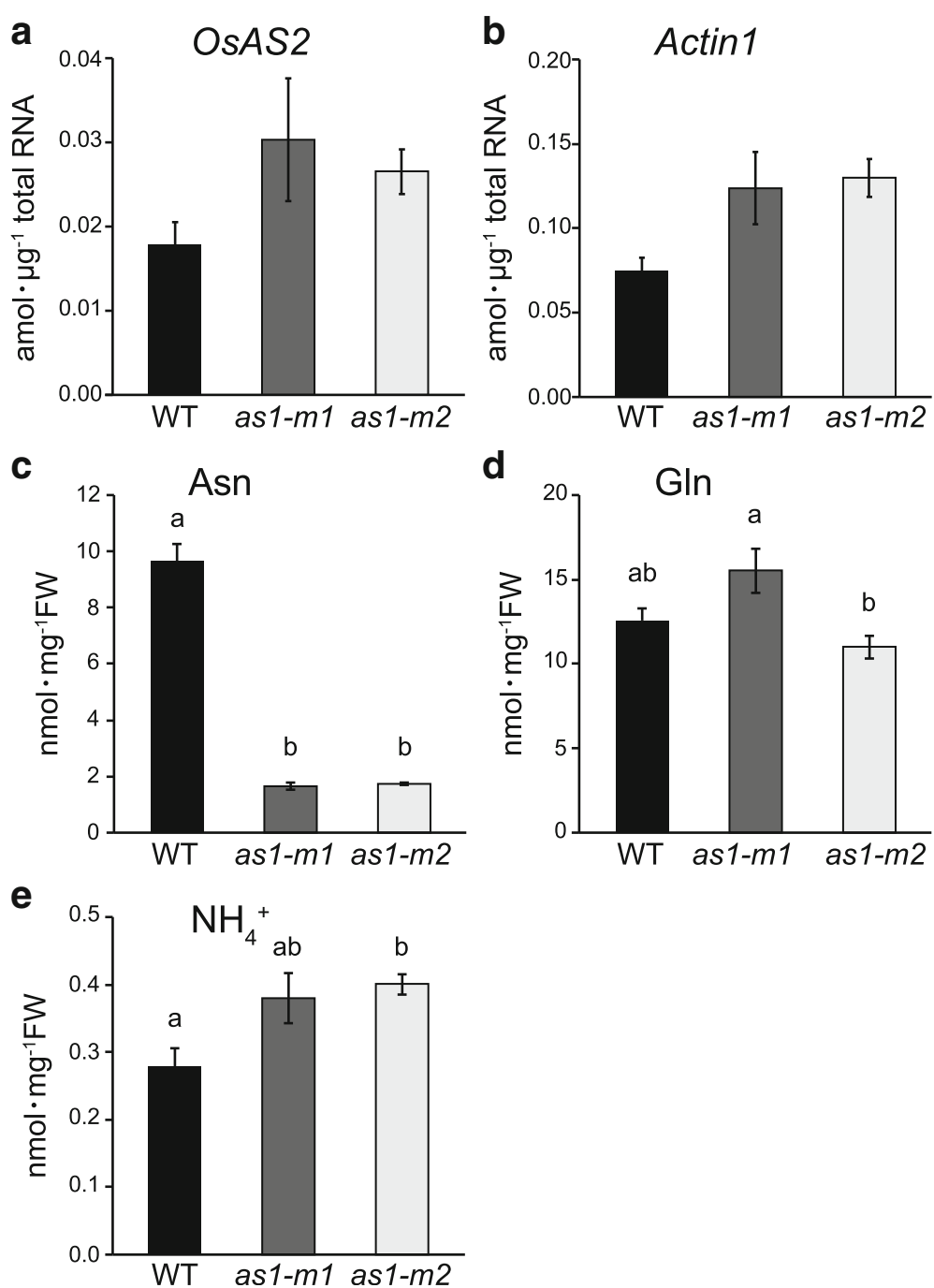

Fig. 6 qPCR and amino acid analysis in the basal portions of wild-type and as1 mutant shoots. QPCR analysis of OsAS2 (a) and Actin1 (b) and analyses of asparagine (c), glutamine (d) and $\mathrm{NH}_{4}{ }^{+}$contents (e) were performed in the basal portions of shoots of the wild type (WT: black column) and two lines of as 1 mutants (as 1-m1 and as 1-m2: dark and light grey column, respectively). Seedlings were grown hydroponically in the presence of $1 \mathrm{mM} \mathrm{NH}_{4} \mathrm{Cl}$ until the fourth-leaf stage. Mean values with $\mathrm{SE}$ of four independent samples are shown. One-way ANOVAs followed by Bonferroni tests were used to identify significant differences between the wild type and as 1 mutants $(P<0.05)$

plants, i.e., in rice, ammonium transporter1;1 (OsAMT1;1) and OsAMT1;2 (Sonoda et al. 2003), NADH-glutamate synthase1 (Hirose et al. 1997), and adenosine phosphateisopentenyltransferases4 (IPT4) and IPT5 (Kamada-Nobusada et al. 2013); in Arabidopsis, glutamine symtnetase1 (Oliveira and Coruzzi 1999), AtAMT1;1 (Rawat et al. 1999), and AtAS (Lam et al. 1994); and nitrate reductase in tobacco leaves (Vincentz et al. 1993). The signalling mechanism in response to glutamine apparently contributes to modulating plant growth. However, few details are known regarding glutamine signalling, unlike the case of nitrate signalling (Sakakibara et al. 2006). It is worth examining the glutamine responsive induction of OsAS1 to understand the mechanism of glutamine signalling in response to $\mathrm{NH}_{4}{ }^{+}$.

As discussed previously (Ohashi et al. 2015b), GS1;2 has a role in the re-assimilation of $\mathrm{NH}_{4}{ }^{+}$released from the phenylalanine ammonia-lyase (PAL) reaction during lignification in the basal portions of shoots. In fact, the biosynthesis of lignin accounts for $10-23 \%$ in rice internodes (Ookawa and Ishihara 1993; Ookawa et al. 1993), and PAL activity in the basal portions of rice shoots was approximately 20-fold higher than in leaf blade at the seedling stage (Additional file 6: Figure S6). Thus, in the basal portions of rice shoots, $\mathrm{NH}_{4}{ }^{+}$released during lignification must be re-assimilated immediately to avoid 

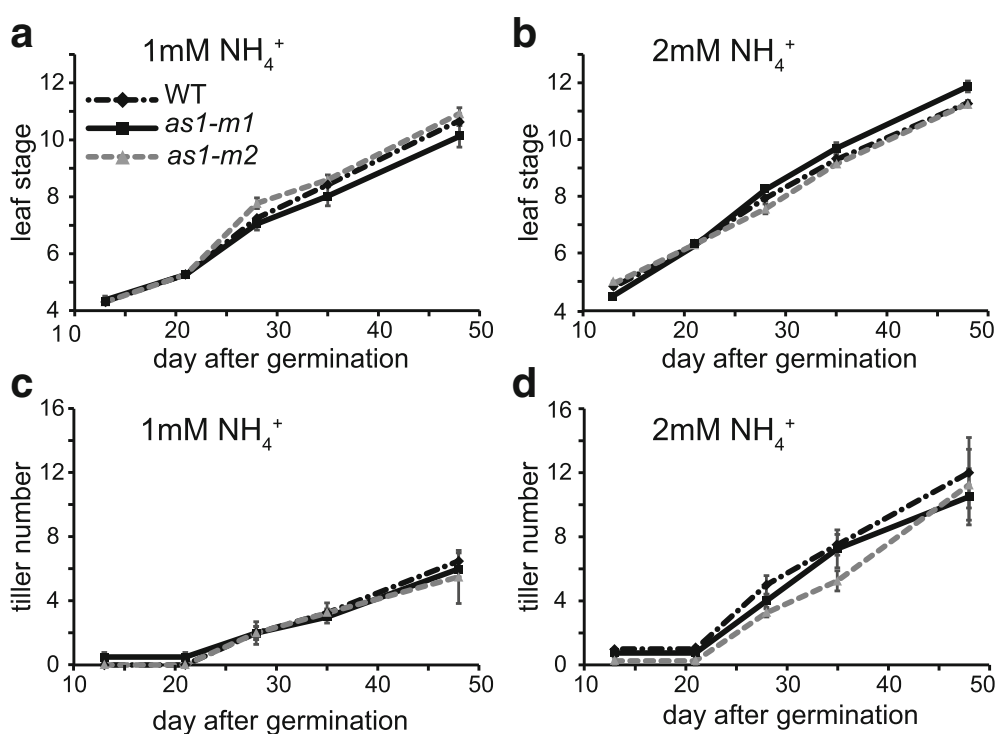

Fig. 7 Comparison of leaf stage and tiller number between the wild type and as 1 mutants. Wild-type (WT: black diamond) plants and two lines of as 1 mutants (as 1- $m 1$ and as 1-m2: black square and grey triangle, respectively) were grown hydroponically in the presence of $1 \mathrm{mM}(\mathbf{a}, \mathbf{c})$ or $2 \mathrm{mM} \mathrm{NH}_{4} \mathrm{Cl}(\mathbf{b}, \mathbf{d})$ until 50 days after germination. Time course studies on measurements of leaf stage and tiller number were conducted. Mean values with SE of four independent samples are shown. One-way ANOVAs followed by Bonferroni tests were used to identify significant differences between the wild type and as 1 mutants $(P<0.05)$

the potential toxicity of $\mathrm{NH}_{4}{ }^{+}$and the loss of nitrogen (Sakurai et al. 2001). In the basal portions of shoots, AS1 and GS1;2 could contribute to the re-assimilation of $\mathrm{NH}_{4}^{+}$during lignification. Considering its cellular localization in phloem companion cells of the nodal vascular anastomoses, the asparagine from AS1 reactions in these cells is apparently transported to sink organs, such as axillary buds, for its outgrowth and development (Fig. 2). Asparagine is catabolized by asparaginase into aspartate as the common precursor of the essential amino acids (Azevedo et al. 2006; Lea et al. 2007; Yabuki et al. 2017).

The lack of GS1;2 caused decreases in tiller number, rice yields and glutamine and asparagine content in the

Table 1 Tiller number, panicle number and parameters of growth of plants growing in the paddy field

\begin{tabular}{lccc}
\hline & wild type & as 1-m1 & as 1-m2 \\
\hline Tiller number per plant & $25.7 \pm 1.83$ & $25.35 \pm 1.01$ & $22.35 \pm 0.49$ \\
Panicle number per plant & $a$ & $a$ & a \\
Shoot length (cm) & $20.32 \pm 1.19$ & $20.55 \pm 0.71$ & $18.66 \pm 0.98$ \\
Shoot dry weight per plant (g) & $57.69 \pm 4.47$ & $65.35 \pm 1.79$ & $50.76 \pm 3.13$ \\
& $100.9 \pm 2.23$ & $100.65 \pm 1.62$ & $94.1 \pm 0.99$ \\
& $a b$ & $a$ & $b$ \\
\hline
\end{tabular}

basal portions of shoots (Funayama et al. 2013; Ohashi et al. 2015b, 2017), while a lack of AS1 caused a decrease in only asparagine content and had less of an effect on tiller number and glutamine content (Figs. 6, 7, Table 1). These results indicate that the availability of asparagine generated from AS1 reactions apparently contributes less to the outgrowth of tillers in rice plants under conditions of sufficient glutamine. Thus, we conclude that the reduction in tiller number in gs 1;2 mutants is independent of the low availability of asparagine in the basal portions of shoots. Previous research has revealed that glutamine generated from GS1;2 reactions contributes to the outgrowth of tillers via glutamine-dependent cytokinin synthesis (Ohashi et al. 2017). Compared to that of glutamine, the contribution of asparagine to the outgrowth of rice tillers is suggested to be limited.

\section{Conclusion}

Our results demonstrated that the expression of OsAS1 could be induced by glutamine or its related metabolite in both roots and the basal portions of rice shoots. Especially in phloem companion cells of the nodal vascular anastomoses, asparagine synthesis is largely dependent on glutamine-responsive AS1. Thus, we conclude that the low availability of asparagine in $g s 1 ; 2$ mutants was caused by the reduction in glutamine content, which is required for the up-regulation of OsAS1 expression. Availability of glutamine is suggested to be more important than that of asparagine for the outgrowth of rice tillers. 


\section{Methods}

\section{Plant Materials}

This study utilized the rice (Oryza. sativa L.) cultivar 'Nipponbare' as the wild-type plants as well as a retrotransposon Tos17-inserted line of the gs1;2 mutant and two lines of as1 mutants (Funayama et al. 2013; Ohashi et al. 2015a). The seeds of these rice plants were germinated and grown hydroponically until either the fourthor seventh-leaf stage in an outdoor greenhouse. The temperature was controlled at $26{ }^{\circ} \mathrm{C}$ during the day with supplemental light for $13 \mathrm{~h}$. The hydroponic culture solution was renewed once per week with $1 \mathrm{mM} \mathrm{NH}_{4} \mathrm{Cl}$ as described in Ohashi et al. (2017). Longitudinal sections $(5 \mathrm{~mm})$ of basal portions of shoots including axillary buds, internodes and a SAMs were prepared by removing the primary and secondary leaves, seeds and roots as described in Ohashi et al. (2017). The basal portions of shoots at the fourth-leaf stage or seventh-leaf stage were used for the determination of the amino acid and $\mathrm{NH}_{4}{ }^{+}$content, in situ hybridization, and qPCR analyses.

When the short-term effects of $\mathrm{NH}_{4}{ }^{+}$supply to roots on gene expression and determination of the free amino acid and $\mathrm{NH}_{4}{ }^{+}$content were tested, the seedlings at the fourthleaf stage or seventh-leaf stage were further grown in water for $3 \mathrm{~d}$ to deplete nitrogen and were then treated with or without $1 \mathrm{mM} \mathrm{NH}_{4} \mathrm{Cl}$ for $8 \mathrm{~h}$ or $24 \mathrm{~h}$ (Ohashi et al. 2017). In MSX-treatment experiments, the seedlings at the fourth-leaf stage grown in water for $3 \mathrm{~d}$ were pretreated with or without $1 \mathrm{mM}$ MSX (Sigma-Aldrich Japan, Tokyo, Japan) for $2 \mathrm{~h}$ and then transferred to media containing no nitrogen, $1 \mathrm{mM} \mathrm{NH}_{4} \mathrm{C} 1$ or $5 \mathrm{mM}$ glutamine for $8 \mathrm{~h}$ according to Kamada-Nobusada et al. (2013) with slight modifications.

For measuring the tiller number, panicle number, shoot length and shoot dry weight at a paddy field, rice plants were grown in the paddy field of the Institute for Sustainable Agro-ecosystem Services in University of Tokyo, Japan (35'44' N, $139^{\circ} 32^{\prime} \mathrm{E}, 60 \mathrm{~m}$ altitude), during the summer (May to October) in 2015 under normal fertilizer, light irradiance and temperature. A germinated seed was sown into each cell of a cell tray and grown in a greenhouse under natural light conditions for four weeks. One seedling was then transplanted into each hill at $18 \mathrm{~cm}$ between hills and $30 \mathrm{~cm}$ between rows. Each plot was composed of four rows with ten hills with four replicates. Commercial compound fertilizer including $\mathrm{N}, \mathrm{P}_{2} \mathrm{O}_{5}$ and $\mathrm{K}_{2} \mathrm{O}$ was used to fertilize at 60,90 , and $80 \mathrm{~kg} \mathrm{ha}^{-1}$ as a basal fertilizer before transplanting. At the panicle initiation stage, tiller numbers of five average plants selected from each plot were measured. After panicle ripening, ten plants in each plot were harvested and shoot length, shoot dry weight without panicle, and panicle number were measured.

\section{qPCR Analysis}

The qPCR analysis was performed according to Konishi et al. (2014). The nucleotide sequences for OsAS1, OsAS2, OsGS1;1, OsGS1;2 and Actin1 are registered in the Rice Annotation Project Database (RAP-DB; http://rapdb.dna. affrc.go.jp/) with accession numbers Os03g0291500, Os06g0265000, Os02g0735200, Os03g0223400 and Os03g0718100, respectively. Each gene-specific primer for qPCR analyses is shown in Tabuchi et al. (2007) and Ohashi et al. (2015a). The PCR products were amplified from the single-stranded cDNA as the template and quantified using a Light Cycler 480 (Roche Diagnostics K. K., Tokyo, Japan) according to the following program: $10 \mathrm{~s}$ at $95{ }^{\circ} \mathrm{C}$, followed by 50 cycles of $95^{\circ} \mathrm{C}$ for $5 \mathrm{~s}$ and $60{ }^{\circ} \mathrm{C}$ for $34 \mathrm{~s}$. The transcript contents were quantitatively determined using each purified cDNA clone as a calibration standard.

\section{In situ Hybridization of OsAS Genes}

Preparation of RNA probes for OsAS1 and OsAS2 and in situ hybridization analysis for the basal portions of shoots were performed as described in Ohashi et al. (2015a, 2017).

\section{Determination of Glutamine, Asparagine and $\mathrm{NH}_{4}{ }^{+}$ Contents}

Free amino acids and $\mathrm{NH}_{4}{ }^{+}$contents in the basal portions of shoots at the fourth-leaf stage were determined as described in Konishi et al. (2014). Derivatization of amino acids and $\mathrm{NH}_{4}{ }^{+}$was carried out using the AccQ-Tag Ultra Derivatization Kit (Nihon Waters K.K., Tokyo, Japan). AccQ-Tag-labelled derivatives were separated and quantified using an ACQUITY UPLC H-Class with a tunable UV detector (Nihon Waters K.K.).

\section{Analysis of Phenylalanine Ammonia-lyase Activity}

The assay for PAL activity was conducted according to Olsen et al. (2008) with slight modifications. Shoot basal portion tissues (80-170 mg fresh weight) were pre-chilled in liquid nitrogen and then milled with a Tissue Lyser II (Qiagen, K. K., Tokyo, Japan) at $20 \mathrm{~Hz}$ for $2 \mathrm{~min}$. Powdered samples were resolved in 5 volumes of extraction buffer (100 mM Tris- $\mathrm{HCl}$ (pH 8.8) with $12 \mathrm{mM} \beta$ mercaptoethanol) of sample weight, mixed in Tissue Lyser II at $20 \mathrm{~Hz}$ for $2 \mathrm{~min}$, and centrifuged at 16,000 $\mathrm{g}$ for $15 \mathrm{~min}$ at $4{ }^{\circ} \mathrm{C}$. The supernatant was passed through a MicroSpin S-200 HR Column (GE Healthcare Japan, Tokyo, Japan), and the elution as enzyme solution was used for the PAL activity assay. The PAL activity assay was performed at $37{ }^{\circ} \mathrm{C}$ for $60 \mathrm{~min}$ in an assay mixture containing $500 \mu \mathrm{l}$ enzyme extract, $450 \mu \mathrm{l} 100 \mathrm{mM}$ Tris$\mathrm{HCl}(\mathrm{pH} 8.8)$ and $50 \mu \mathrm{l} 100 \mathrm{mM}$ L-phenylalanine. The reaction was terminated by adding $50 \mu \mathrm{l}$ of $5 \mathrm{M} \mathrm{HCl}$, and absorbance was recorded at $290 \mathrm{~nm}$ against blanks made in the same way as the assays but with $50 \mu \mathrm{l}$ of $5 \mathrm{M} \mathrm{HCl}$ 
added before L-phenylalanine. The amount of product formed was calculated from the increase in absorbance using an extinction coefficient for cinnamate of 10,000 L/cm/mole (Zimmerman and Hahlbrock 1975).

\section{Statistics}

All data sets were analysed using Microsoft Excel add-in software (Social Survey Research Information Co., Ltd., Tokyo, Japan).

\section{Additional files}

Additional file 1: Figure S1. qPCR analysis of OsGS1;1 and OsGS1;2 in the basal portions of wild-type shoots using MSX. Wild-type rice seedlings were grown hydroponically in the presence of $1 \mathrm{mM} \mathrm{NH}_{4} \mathrm{Cl}$ until the fourth-leaf stage and then in water for $3 \mathrm{~d}$. After pre-treatment with $1 \mathrm{mM} \mathrm{MSX}$ for $2 \mathrm{~h}$, the seedlings were treated for $8 \mathrm{~h}$ with $1 \mathrm{mM} \mathrm{NH}_{4}^{+}$ $\left(\mathrm{MSX}+\mathrm{NH}_{4}^{+}\right)$or $5 \mathrm{mM}$ glutamine $(\mathrm{MSX}+\mathrm{Gln})$. The seedlings without MSX pre-treatment were also treated for $8 \mathrm{~h}$ with $1 \mathrm{mM} \mathrm{NH}_{4}{ }^{+}\left(+\mathrm{NH}_{4}{ }^{+}\right)$, $5 \mathrm{mM}$ glutamine $(\mathrm{Gln})$ or without nitrogen nutrients $(-\mathrm{N})$. QPCR analysis of OsGS1;1 (a) and OsGS1;2 (b) were performed in the shoot basal parts. Fold changes of transcript levels in each treatment relative to those in -N were calculated, and mean values with SE of four independent samples are shown. Asterisks denote statistically significant differences between samples treated without $1 \mathrm{mM} \mathrm{NH}_{4}{ }^{+}$and each treated sample (*, $P<0.05$ by Student's $t$-test). (EPS $1807 \mathrm{~kb}$ )

Additional file 2: Figure S2. qPCR analysis of OSAS1 and OSAS2 in roots of wild-type rice using MSX. Wild-type rice seedlings were grown hydroponically in the presence of $1 \mathrm{mM} \mathrm{NH}_{4} \mathrm{Cl}$ until the fourth-leaf stage and then transferred into water for $3 \mathrm{~d}$. After pre-treatment with $1 \mathrm{mM}$ MSX for $2 \mathrm{~h}$, the seedlings were treated for $8 \mathrm{~h}$ with $1 \mathrm{mM} \mathrm{NH}_{4}{ }^{+}$(MSX + $\mathrm{NH}_{4}{ }^{+}$) or $5 \mathrm{mM}$ glutamine (MSX $+\mathrm{Gln}$ ). The seedlings without MSX pre-treatment were also treated for $8 \mathrm{~h}$ with $1 \mathrm{mM} \mathrm{NH}_{4}{ }^{+}\left(+\mathrm{NH}_{4}{ }^{+}\right), 5 \mathrm{mM}$ glutamine (Gln) or without nitrogen nutrients $(-\mathrm{N})$. qPCR analyses of OsAS1 (a), OsAS2 (b), and actin1 transcripts (c) were performed in roots. Fold changes of transcript levels in each treatment relative to those in $-\mathrm{N}$ were calculated, and mean values with SE of four independent samples are shown. Asterisks denote statistically significant differences between samples treated without $1 \mathrm{mM} \mathrm{NH}_{4}{ }^{+}$and each treated sample $\left({ }^{*}, P<0.05\right.$ by Student's t-test). (EPS $1890 \mathrm{~kb}$ )

Additional file 3: Figure S3. Amino acid analysis in the basal portions of shoots supplied with transient $\mathrm{NH}_{4}{ }^{+}$. Seedlings of the wild type (WT: black column) and two lines of as 1 mutants (as 1-m1 and as 1-m2: dark and light grey column, respectively) at the fourth-leaf stage were grown in water for $3 \mathrm{~d}$, then treated with $\left(+\mathrm{NH}_{4}{ }^{+}\right)$or without (-N) $1 \mathrm{mM} \mathrm{NH}_{4} \mathrm{Cl}$ for $24 \mathrm{~h}$. Mean values with SE of four independent samples are shown. Oneway ANOVAs followed by Bonferroni tests were used to identify significant differences between the WT and as 1 mutants $(P<0.05)$. (EPS $1925 \mathrm{~kb}$ )

Additional file 4: Figure S4. Stereoscopic microscope observation of the primary tiller at fifth leaf stage. Seedlings of the wild type (WT) (a) and two lines of as 1 mutants (as 1-m1 and as 1-m2) (b, c) were grown hydroponically in the presence of $1 \mathrm{mM} \mathrm{NH}_{4}^{+}$until the fifth leaf stage. The primary tiller was observed by microscope. Scale bars $=2 \mathrm{~mm}$. (JPG $1217 \mathrm{~kb}$ )

Additional file 5: Figure S5. Photos of wild type and as 1 mutants at heading stage. Wild-type plants (WT) (a) and two lines of as 1 mutants (as 1-m1 and as 1-m2) (b, c) were grown in the paddy field under normal fertilization. Each two plants were shown. (JPG 1956 kb)

Additional file 6: Figure S6. Comparison of the PAL activity between basal portions of shoots and the third-leaf blade. Rice seedlings were grown hydroponically in the presence of $1 \mathrm{mM} \mathrm{NH}_{4} \mathrm{Cl}$ until the fourth-leaf stage, and the basal portions of shoots (black column) and third-leaf blades (grey column) were harvested. Mean values with SE of five independent samples are shown. Asterisks denote statistically significant differences between each organ $\left({ }^{*}, P<0.05\right.$ by Student's t-test). (EPS $1857 \mathrm{~kb}$ )

\section{Abbreviations}

AMT: Ammonium transporter; AS: Asparagine synthetase; GS: Glutamine synthetase; IPT: Adenosine phosphate-isopentenyltransferase; MSX: Methionine sulfoximine; PAL: Phenylalanine ammonia-lyase; qPCR: Quantitative real-time PCR; SAM: Shoot apical meristem

\section{Acknowledgements}

We would like to express our deep gratitude to the technical support staff of the Institute for Sustainable Agro-ecosystem Services (ISAS) at the University of Tokyo for their help in the cultivation and management of rice. We thank Drs. Hirohiko Hirochika and Akio Miyao, The Project for Rice Genome Research, and The National Institute of Agrobiological Sciences, Tsukuba, Japan, for providing seeds of Tos17-inserted rice mutants. We would like to thank American Journal Experts (www.aje.com) for English language editing.

\section{Funding}

This work was supported by the Ministry of Education, Culture, Sports, Science and Technology of Japan [a Grant-in-Aid for Scientific Research on Innovative Area (22119003 to TY) and a GRENE NC-CARP project]; the Japan Society for the Promotion of Science (JSPS) KAKENHI [Grant No. 15 K14674 and 15 H04468 to TH, 26450073 to SK, and 16 J01799 to MO]; and the Division for International Advanced Research and Education (DIARE), Tohoku University.

\section{Availability of Data and Materials}

The datasets supporting the conclusions of this article are included within the article and its additional files.

\section{Authors' Contributions}

$\mathrm{MO}$ and $\mathrm{KI}$ designed this study, performed most of the experiments and wrote the manuscript. NK operated UPLC for amino acid analysis. KS conducted the paddy field trials. SK, MM, TH and TY advised and assisted in the design of this study and in writing the manuscript. All authors read and approved the final manuscript.

\section{Ethics Approval and Consent to Participate}

Not applicable.

\section{Competing Interests}

The authors declare that they have no competing interests.

\section{Publisher's Note}

Springer Nature remains neutral with regard to jurisdictional claims in published maps and institutional affiliations.

\section{Author details}

'Graduate School of Agricultural Science, Tohoku University, 468-1 Aoba, Aramaki-Aza, Aoba-ku, Sendai 980-8572, Japan. ${ }^{2}$ The University of Tokyo, Graduate School of Agricultural and Life Sciences, Institute of Sustainable Agro-ecosystem Services (ISAS), 1-1-1 Midori-cho, Nishitokyo, Tokyo 188-0002, Japan. ${ }^{3}$ Present Address: Graduate School of Bioagricultural Sciences, Nagoya University, Furo-cho, Chikusa-ku, Nagoya 464-8601, Japan. ${ }^{4}$ Present Address: Institute of Plant Science and Resources, Okayama University, 2-20-1 Chuo, Kurashiki 710-0046, Japan. ${ }^{5}$ Present Address: Division for Interdisciplinary Advanced Research and Education, Tohoku University, 6-3 Aoba, Aramaki-Aza, Aoba-ku, Sendai 980-0845, Japan.

Received: 10 December 2017 Accepted: 30 April 2018 Published online: 09 May 2018

\section{References}

Azevedo RA, Lancien M, Lea PJ (2006) The aspartic acid metabolic pathway, an exciting and essential pathway in plants. Amino Acids 30:143-162

Domagalska MA, Leyser O (2011) Signal integration in the control of shoot branching. Nat Rev Mol Cell Biol 12:211-221

Evers JB, van der Krol AR, Vos J, Struik PC (2011) Understanding shoot branching by modelling form and function. Trends Plant Sci 16:464-467

Fukumorita T, Chino M (1982) Sugar, amino-acid, and inorganic contents in rice phloem sap. Plant Cell Physiol 23:273-283 
Funayama K, Kojima S, Tabuchi-Kobayashi M, Sawa Y, Nakayama Y, Hayakawa T, Yamaya T (2013) Cytosolic glutamine synthetase1;2 is responsible for the primary assimilation of ammonium in rice roots. Plant Cell Physiol 54:934-943

Gaufichon L, Reisdorf-Cren M, Rothstein SJ, Chardon F, Suzuki A (2010) Biological functions of asparagine synthetase in plants. Plant Sci 179:141-153

Hayashi H, Chino M (1990) Chemical composition of phloem sap from the upper most internode of the rice plant. Plant Cell Physiol 31:247-251

Hirose N, Hayakawa T, Yamaya T (1997) Inducible accumulation of mRNA for $\mathrm{NADH}$-dependent glutamate synthase in rice roots in response to ammonium ions. Plant Cell Physiol 38:1295-1297

Hoshikawa K (1989) The growing Rice Plant. Nosan Gyoson Bunka Kyokai, Tokyo Ireland RJ, Lea PJ (1999) The enzymes of glutamine, glutamate, asparagine, and aspartate metabolism. In: Singh BK (ed) In plant amino acids: biochemistry and biotechnology. Marcel Dekker, Inc, New York, pp 49-109

Ishiyama K, Inoue E, Tabuchi M, Yamaya T, Takahashi H (2004) Biochemical background and compartmentalized functions of cytosolic glutamine synthetase for active ammonium assimilation in rice roots. Plant Cell Physiol 45:1640-1647

Kamada-Nobusada T, Makita N, Kojima M, Sakakibara H (2013) Nitrogen-dependent regulation of de novo cytokinin biosynthesis in rice: the role of glutamine metabolism as an additional signal. Plant Cell Physiol 54:1881-1893

Kawachi T, Sueyoshi K, Nakajima A, Yamagata H, Sugimoto T, Oji Y (2002) Expression of asparagine synthetase in rice (Oryza sativa) roots in response to nitrogen. Physiol Plant 114:41-46

Konishi N, Ishiyama K, Matsuoka K, Maru I, Hayakawa T, Yamaya T, Kojima S (2014) $\mathrm{NADH}$-dependent glutamate synthase plays a crucial role in assimilating ammonium in the Arabidopsis root. Physiol Plant 152:138-151

Lam HM, Peng SS, Coruzzi GM (1994) Metabolic regulation of the gene encoding glutamine-dependent asparagine synthetase in Arabidopsis thaliana. Plant Physiol 106:1347-1357

Lea PJ, Miflin BJ (1974) Alternative route for nitrogen assimilation in higher plants. Nature 251:614-616

Lea PJ, Sodek L, Parry MAJ, Shewry PR, Halford NG (2007) Asparagine in plants. Ann Appl Biol 150:1-26

Liu Y, Gu D, Ding Y, Wang Q, Li G, Wang S (2011) The relationship between nitrogen, auxin and cytokinin in the growth regulation of rice (Oryza sativa $\mathrm{L}$. ) tiller buds. Aust J Crop Sci 5:1019-1026

Mae T (1997) Physiological nitrogen efficiency in rice: nitrogen utilization, photosynthesis, and yield potential. Plant Soil 196:201-210

Ohashi M, Ishiyama K, Kojima S, Kojima M, Sakakibara H, Yamaya T, Hayakawa T (2017) Lack of cytosolic glutamine synthetase 1;2 activity reduces nitrogendependent biosynthesis of cytokinin required for axillary bud outgrowth in rice seedlings. Plant Cell Physiol 58:679-690

Ohashi M, Ishiyama K, Kojima S, Konishi N, Nakano K, Kanno K, Hayakawa T, Yamaya T (2015a) Asparagine synthetase1, but not asparagine synthetase2, is responsible for the biosynthesis of asparagine following the supply of ammonium to rice roots. Plant Cell Physiol 56:769-778

Ohashi M, Ishiyama K, Kusano M, Fukushima A, Kojima S, Hanada A, Kanno K, Hayakawa T, Seto Y, Kyozuka J, Yamaguchi S, Yamaya T (2015b) Lack of cytosolic glutamine synthetase 1;2 in vascular tissues of axillary buds caused severe reduction in their outgrowth and disorder of metabolic balance in rice seedlings. Plant J 81:347-356

Oliveira IC, Coruzzi GM (1999) Carbon and amino acids reciprocally modulate the expression of glutamine synthetase in Arabidopsis. Plant Physiol 121:301-310

Olsen KM, Lea US, Slimestad R, Verheul M, Lillo C (2008) Differential expression of four Arabidopsis PAL genes; PAL1 and PAL2 have functional specialization in abiotic environmental-triggered flavonoid synthesis. J Plant Physiol 165:1491-1499

Ookawa T, Ishihara K (1993) Varietal difference of the cell wall components affecting the bending stress of the culm in relation to the loading resistance in paddy rice. Jpn J Crop Sci 62:378-384

Ookawa T, Todokoro Y, Ishihara K (1993) Changes in physical and chemical characteristics of culm associated with lodging resistance in paddy rice under different growth conditions and varietal difference of their changes. Jpn J Crop Sci 62:525-533

Rawat SR, Silim SN, Kronzucker HJ, Siddiqi MY, Glass AD (1999) AtAMT1 gene expression and $\mathrm{NH}_{4}{ }^{+}$uptake in roots of Arabidopsis thaliana: evidence for regulation by erroot glutamine levels. Plant J 19:143-152

Sakakibara H, Takei K, Hirose N (2006) Interactions between nitrogen and cytokinin in the regulation of metabolism and development. Trends Plant Sci 11:440-448
Sakamoto T, Matsuoka M (2008) Identifying and exploiting grain yield genes in rice. Curr Opin Plant Biol 11:209-214

Sakurai N, Katayama Y, Yamaya T (2001) Overlapping expression of cytosolic glutamine synthetase and phenylalanine ammonia-lyase in immature leaf blades of rice. Physiol Plant 113:400-408

Sonoda Y, Ikeda A, Saiki S, von Wirén N, Yamaya T, Yamaguchi J (2003) Distinct expression and function of three ammonium transporter genes (OsAMT1;1-1; 3) in rice. Plant Cell Physiol 44:726-734

Tabuchi M, Abiko T, Yamaya T (2007) Assimilation of ammonium ions and reutilization of nitrogen in rice (Oryza sativa L.). J Exp Bot 58:2319-2327

Umehara M, Hanada A, Yoshida S, Akiyama K, Arite T, Takeda-Kamiya N, Magome H, Kamiya Y, Shirasu K, Yoneyama K, Kyozuka J, Yamaguchi S (2008) Inhibition of shoot branching by new terpenoid plant hormomes. Nature 455:195-200

Urquhart AA, Joy KW (1981) Use of phloem exudate technique in the study of amino acid transport in pea plants. Plant Physiol 68:750-754

Vincentz M, Moureaux T, Leydecker MT, Vaucheret H, Caboche M (1993) Regulation of nitrate and nitrite reductase expression in Nicotiana plumbaginifolia leaves by nitrogen and carbon metabolites. Plant J 3:315-324

Yabuki Y, Ohashi M, Imagawa F, Ishiyama K, Beier MP, Konishi N, Umetsu-Ohashi T, Hayakawa T, Yamaya T, Kojima S (2017) A temporal and spatial contribution of asparaginase to asparagine catabolism during development of rice grains. Rice 10. https://doi.org/10.1186/s12284-017-0143-8

Yamaya T, Oaks A (2004) Metabolic regulation of ammonium uptake and assimilation. In: Amâncio S, Stulen I (eds) In nitrogen acquisition and assimilation in higher plants. Kluwer Academic Publishers, Dordrecht, pp 35-63

Zimmerman A, Hahlbrock K (1975) Light induced changes in enzyme activities in parsley cell suspension. Purification and some properties of phenylalanine ammonia lyase. Arch Biochem Biophys 166:54-62

\section{Submit your manuscript to a SpringerOpen ${ }^{\mathcal{O}}$ journal and benefit from:}

- Convenient online submission

- Rigorous peer review

- Open access: articles freely available online

- High visibility within the field

- Retaining the copyright to your article

Submit your next manuscript at $>$ springeropen.com 